\title{
Solution precursor plasma spraying (SPPS): a novel and simple process to obtain bioactive glass coatings
}

\author{
E. Cañas ${ }^{(a, *)}$, M.J. Orts ${ }^{(a)}$, A.R. Boccaccini ${ }^{(b)}$, E. Sánchez ${ }^{(a)}$ \\ a) Instituto de Tecnología Cerámica (ITC), Universitat Jaume I (UJI), 12006, Castellón, Spain \\ b) Institute of Biomaterials, University of Erlangen-Nuremberg, 91058, Erlangen, Germany
}

Eugeni Cañas Recacha

Email: eugeni.canas@itc.uji.es

María José Orts Tarí

Email: mariajose.orts@itc.uji.es

Aldo R. Boccaccini

Email: aldo.boccaccini@fau.de

Enrique Sánchez Vilches

Email: enrique.sanchez@itc.uji.es 


\section{Abstract}

This research addresses the use of an emerging plasma spray technique known as SPPS (Solution Precursor Plasma Spraying) to obtain bioactive glass coatings. In SPPS, the feedstock is a sol precursor of the bioactive glass instead of glass particles. Precursor feedstock composition (presence or not of acid catalyst), plasma torch enthalpy and stand-off distance of the spraying process have been varied to produce bioactive glass coatings of nominally 45S5 composition. A good quality coating was obtained when catalyst ( $0.2 \mathrm{M}$ acid nitric) was used in the precursor and the plasma spraying took place with shorter stand-off distance and higher plasma enthalpy. Bioactivity in Simulated Body Fluid test was confirmed and an effect of the coating interconnected porosity to enhance bioactivity was observed. Keywords: Bioceramics; Solution feedstock; Solution precursor plasma spraying; Bioactive glass coating; Simulated body fluid; Microstructure

\section{Introduction}

Bioactive glasses are considered promising materials to be used as coatings onto metallic substrates in load-bearing applications due to their high bioactivity [1,2]. Among different application techniques, thermal spraying shows significant advantages; in particular, the fact that the deposition and consolidation of the coating occur simultaneously without the need of a sintering treatment. There have been some previous attempts to obtain bioactive glass coatings starting with glass particle feedstocks by different thermal spray techniques. Although coating bioactivity has been proven, the uncompleted sintering of glass particles during the spraying deposition gives rise to a highly porous microstructure poorly adhered to the substrate $[3,4]$. More recently, researchers have tried to use the suspension plasma spraying (SPS) process in which a glass particle suspension is fed into the plasma torch instead of a powder feedstock [5]. Although finer glass particles can be fed with a suspension, similar microstructures to those obtained from powder feedstocks are still produced [5,6]. In addition, regardless the use of a powder or particle suspension as feedstock, glass particles have to be produced by the fusing and crushing process which implies a series of laborious preparation steps such as melting, quenching, grinding, sieving, etc., which can introduce contaminants in the final feedstock.

Nowadays, the use of precursor solutions instead of traditional thermal spraying feedstocks (powders and suspensions) is gaining great interest in the thermal spray community due to the unique properties obtained, i.e thinner and nanostructured coatings with denser and more homogeneous microstructures [7]. 
In addition, the use of precursor solutions allows to obtain high purity feedstocks, avoiding possible contamination from feedstock preparation steps. In the family of thermal spraying techniques, there is a process known as SPPS (Solution Precursor Plasma Spraying) which has been extensively reported for varied materials (zirconia or hydroxyapatite, among others) but it has hardly been investigated with glass precursors [7]. In a previous research, authors have considered the production of bioactive glass coating by SPPS and SPS processes [8]. However, highly crystallized glass-ceramic coatings were obtained when a fixed sol precursor feedstock was used at given plasma spray conditions. The study presented in this letter has focused, for the first time, on the development of fully amorphous, bioactive glass coatings by varying feedstock composition as well as plasma spray variables in an SPPS process.

\section{Experimental}

\subsection{Solution feedstocks}

Bioactive glass of $45 \mathrm{~S} 5$ composition $\left(\mathrm{SiO}_{2} 45 \%, \mathrm{Na}_{2} \mathrm{O} 24.5 \%, \mathrm{CaO} 24.5 \%\right.$, and $\mathrm{P}_{2} \mathrm{O}_{5} 6 \%$, in wt.\%) was selected. Tetraethyl orthosilicate $\left(\mathrm{C}_{8} \mathrm{H}_{20} \mathrm{O}_{4} \mathrm{Si}\right.$ synthesis grade, Merck, Germany) or TEOS, triethyl phosphate $\left(\mathrm{C}_{6} \mathrm{H}_{15} \mathrm{O}_{4} \mathrm{P}\right.$ synthesis grade, Merck, Germany) or TEP, calcium nitrate $\left(\mathrm{Ca}\left(\mathrm{NO}_{3}\right)_{2} \cdot 4 \mathrm{H}_{2} \mathrm{O}>99 \%\right.$, Sigma-Aldrich, USA), and sodium nitrate $\left(\mathrm{NaNO}_{3}>99 \%\right.$, VWR Chemicals, USA) were used as the precursors for $\mathrm{SiO}_{2}, \mathrm{P}_{2} \mathrm{O}_{5}, \mathrm{CaO}$, and $\mathrm{Na}_{2} \mathrm{O}$, respectively.

The sol precursor was prepared in aqueous media at a concentration of $4 \mathrm{M}$ with $0.2 \mathrm{M}$ nitric acid $\left(\mathrm{HNO}_{3}\right.$ Tritripur, Merck, Germany) as catalyst. A precursor without catalyst was also prepared. Higher amounts of catalyst were not used because it would shorten the gelation time. First, TEOS and deionized water were mixed under magnetic stirring, with a molar ratio of TEOS to water of 1:18, and the necessary amount of $\mathrm{HNO}_{3}$ (if required) was slowly added to the solution, keeping stirring until solution clarification. Then, TEP was added to the solution under stirring $30 \mathrm{~min}$ later, and then calcium and sodium nitrates after another $30 \mathrm{~min}$. The solution was kept stirring for an additional hour and the resulting sol was then aged overnight at room temperature before being used as feedstock.

\subsection{Coating deposition and characterisation}

Coating deposition was carried out in a thermal spraying facility comprised by a plasma torch (F4MB, Oerlikon Metco, Switzerland) coupled to a six axes robot (IRB 1400, ABB, Switzerland) and a home-made liquid feeding system operated by mechanical injection. More details about this facility have been reported elsewhere [9]. 
The substrates employed were discs with a diameter of $25 \mathrm{~mm}$ made of AISI type 304 stainless steel, which were grit-blasted using black corundum and then cleaned with ethanol prior to the deposition. In addition, all substrates were coated by powder plasma spraying with a $\mathrm{TiO}_{2}$ bond coat (Metco 102, Oerlikon Metco, Switzerland) to enhance top coat adherence (Table 1). Finally, precursor glass coatings were deposited by SPPS on preheated, bond coated substrates $\left(300{ }^{\circ} \mathrm{C}-350{ }^{\circ} \mathrm{C}\right)$ to further adherence enhancement. Argon was used as primary plasma gas and hydrogen as secondary. The spraying conditions employed are detailed in Table 1. The following spraying conditions were varied: solution feedstock (with and without catalyst), ratio of plasma gases $\left(\mathrm{Ar} / \mathrm{H}_{2}\right)$ giving rise to different torch enthalpy and stand-off distance. Modification of these three variables led to an 8 experiments grid.

Table 1. Spraying conditions for bond and top coats for the 8 experiments carried out

\begin{tabular}{lcc}
\hline & Bond coat $\left(\mathbf{T i O}_{2}\right)$ & Bioactive glass coating \\
\hline Argon flow rate (slpm*) & 38 & $25 / 45$ \\
Hydrogen flow rate (slpm*) & 14 & 15 \\
Arc intensity (A) & 600 & 600 \\
Stand-off distance (mm) & 120 & $70 / 110$ \\
Number of torch scans & 2 & 5 \\
Torch scan velocity (mm/s) & 1000 & 1250 \\
Injector diameter (mm) & 1.80 & 0.15 \\
Powder flow rate (g/min) & 45 & - \\
Solution flow rate (mL/min) & - & 33 \\
\hline
\end{tabular}

*slpm: standard litres per minute

Coatings were analysed by X-ray diffraction (XRD) using a diffractometer (Advance diffractometer, Bruker Theta-theta, Germany) and were observed in both surface and cross-section by field emission gun environmental scanning electron microscopy (FEG-ESEM) (Quanta 200FEG, FEI Company, USA). Moreover, the coatings were immersed in Simulated Body Fluid (SBF) to investigate their bioactive character according to a standard protocol [10], employing soaking times ranging from 1 to 7 days.

\section{Results and discussion}

In figure 1 , for experiments 1 to 4 (without catalyst), only small discontinuous regions covered by bioactive glass particles with very poor attachment to the substrate were obtained. Hence, without adding catalyst, the sol-gel process and consequently the glass network forming hardly occur in the plasma torch. For this reason, a continuous coating layer is not obtained. This finding contrast with other SPPS processes in which no catalyst was used and coating layers could still be formed [11]. 

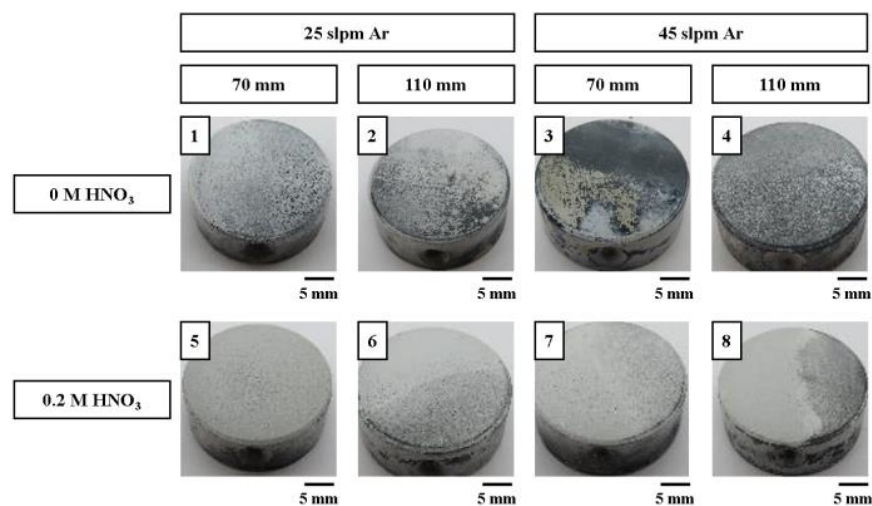

Figure 1. Macroscopic views of the coatings obtained from the eight deposition experiments On the contrary, experiments 5 to 8 (with catalyst) succeeded in obtaining rather uniform coatings although the quality of the surfaces was very different. Thus, trials 5 and 7 led to better quality surfaces because of the shorter stand-off distances used. As reported elsewhere [5], shorter stand-off distances give rise to hotter substrates during deposition which allows glass molten particles to adhere onto the substrate. Finally, the best quality layer was obtained in experiment 5 which combines shorter stand-off distance and higher plasma torch enthalpy. As expected, higher energy during deposition in an SPPS process contributes to the development of the different physical processes occurring from the solution feeding into the plasma torch to the final glass molten particle deposition, i.e. evaporation, gelation, pyrolysis, sintering and melting of glass particles [11]. In addition, as the stand-off distance and torch power are key factors to provide precursors with enough time to develop all the necessary chemical reactions to form the desired material, the solution with $0.2 \mathrm{M}$ of nitric acid was sprayed using the same parameters employed in experiment 5, collecting in-flight particles before impacting onto the substrate and determining their composition by X-ray fluorescence (XRF). From the above, it was found that the maximum deviation in composition was $0.5 \mathrm{wt} \%$ regarding the nominal one, so the obtained coating has a composition very close to that.

Figure 2 displays the surface and cross-section micrographs of the best quality coating obtained (sample 5). It can be observed that a thin layer of a quite homogeneous coating was produced with an average thickness of $35 \mu \mathrm{m}$. Interconnected porosity (marked P) associated to thermal spray processes is also observable giving rise to a high inner surface area. Nevertheless, this specific surface area can be a favourable feature for bioactivity, as reported in the literature for bioactive glasses [10,12]. From the coating surface study, a "two-zones microstructure" common in SPS can be appreciated with a 
cauliflower-like surface, which results from the high concentration of the solution feedstock [13]. Instead of atomising, the solution was radially injected into the torch with a continuous stream, resulting in droplet fragmentation when they make contact with the plasma torch and solvent evaporation rapidly occurs. This step is followed by a shell formation on the drops surface and the breaking of that shell due to an increment of the vapor pressure inside the drop. Thus, part of the shells and spherical particles tend to combine in-flight resulting in the cauliflower-like agglomerates on the coating surface. In the same figure, the XRD pattern of the coating is included. As observed, the coating shows a fully amorphous nature which indicates that the glass network was formed, and no crystallisation took place during deposition and cooling. Only few peaks, corresponding to the $\mathrm{TiO}_{2}$ bond coat, are observed. This finding contrasts with the results obtained by Y. Xiao et al. [8], who attributed the high degree of crystallisation in the coating to an inhibited droplet breakup due to high viscosity gel which resulted in insufficient melting of the glass droplets. Nevertheless, in that research long stand-off distances and low energy plasma were used for the deposition of the feedstock.


Figure 2. Surface (a) and cross-section (b) micrographs of the coating obtained from sample 5. The XRD pattern is also displayed (c). For the sake of comparison the XRD pattern of the coating after 7 days in SBF is also included (d).

Figure 3 shows the micrographs corresponding to the coating from experiment 5 immersed in SBF.

Figures $3 \mathrm{a}$ and $3 \mathrm{~b}$ show this coating before and after 7 days of immersion, respectively, and hydroxycarbonate apatite (HCA) layer was identified throughout the coating (Figure 3b) following the standard protocol by energy dispersive X-ray (EDX) and XRD [10]. Moreover, from this figure, a 
different reaction behaviour in function of the coating zone can be appreciated. As anticipated above, interconnected porosity favours bioactivity of bioactive glass surfaces, therefore the inner surface (Figure 3c) is "more reacted" than the cauliflower-like splats of the top zone (Figure 3d). Besides, in both cases (Figures $3 \mathrm{e}$ and $3 \mathrm{f}$ ), it is possible to appreciate at very high magnifications the typical HCA flake-like crystals, as reported also in literature [14]. In addition, in figure 2d, a XRD pattern corresponding to the "best" coating (sample 5) after 7 days of immersion in SBF is also included. As compared with the one corresponding to the non-immersed coating, a new crystalline phase started to appear due to the interaction of the coating surface with SBF. As expected, the peaks correspond to hydroxycarbonate apatite. Indeed, it is well known that in SBF the glass surface develops a silica rich layer after ion exchange, on which $\mathrm{Ca}$ and $\mathrm{P}$ ions precipitate forming the HCA layer [10].

From the obtained results, it can be concluded that a well adhered coating was obtained with a suitable bioactivity, being this property highly influenced by the microstructure features of the coating. Therefore, this fact should be considered in the design of SPPS bioactive glass coatings. 


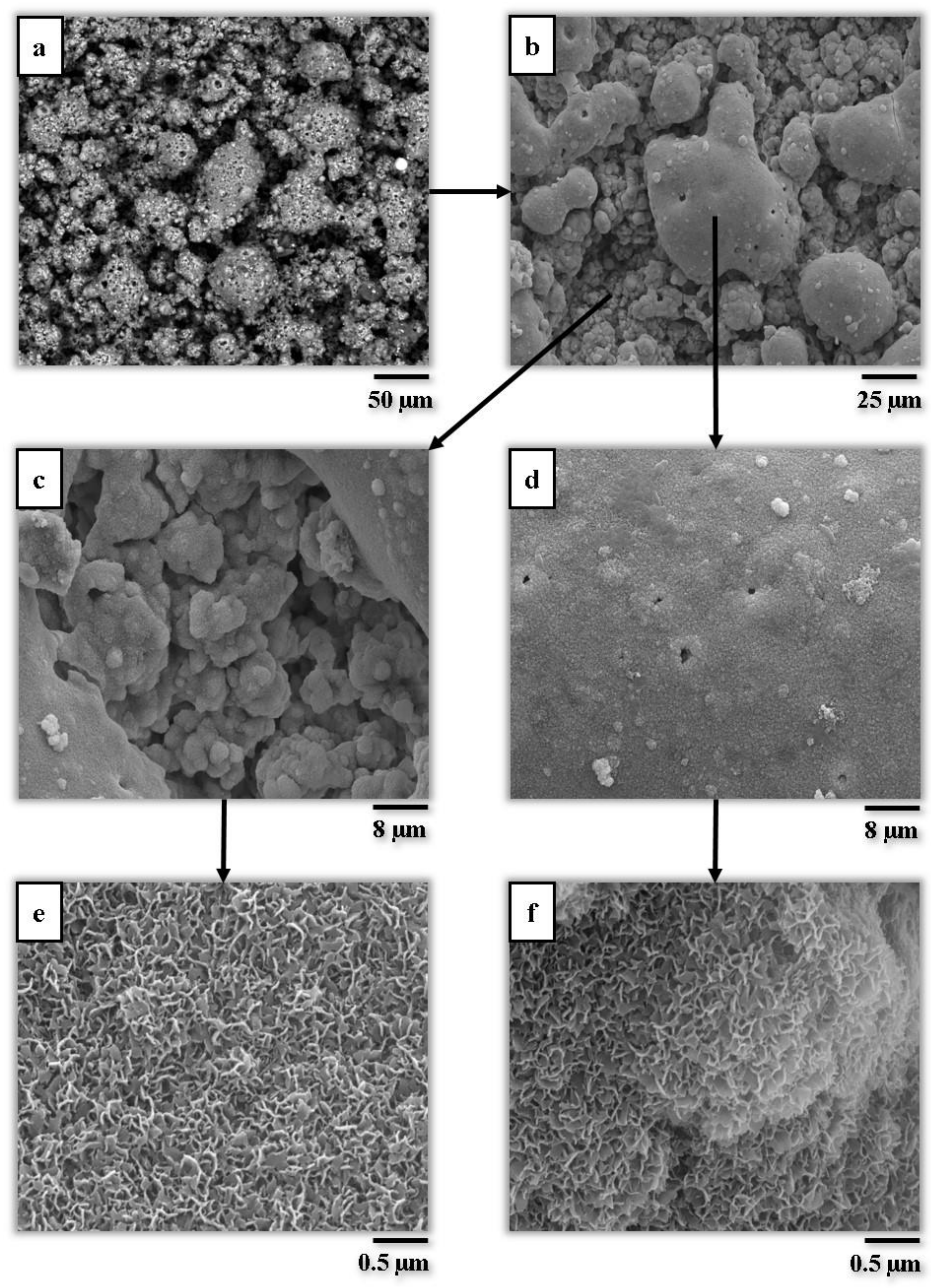

Figure 3. Micrographs of the coating from the experiment 5 after 7 days of immersion in SBF. a) Coating before immersion; b) coating after 7 days in SBF; c) inner zone of the immersed coating; d) top zone of the immersed coating; e) HCA crystals of the inner zone; f) HCA crystals of the top zone

\section{Conclusions}

The SPPS process has been employed to obtain bioactive glass coatings from a sol precursor of the 45S5 bioactive glass composition. A good quality coating has been obtained, which exhibits high surface area and the formation of a HCA layer, when immersed in SBF for 1 week, confirming the bioactivity of this coating.

Based on these results, it can be concluded that SPPS represents a suitable method to produce bioactive glass coatings in a simple way, avoiding the time-consuming steps related to the preparation of glass particles. In addition, the use of a solution precursor can facilitate the incorporation of new doping elements to the feedstock composition, which could lead to bioactive glass coatings with biologically active ions. Consequently, these facts open new research opportunities concerning feedstock design and 
microstructure optimization in the SPPS process to expand the medical applications of bioactive glass coatings.

\section{Acknowledgements}

Authors thank Universitat Jaume I of Castellon for the support provided by funding its Research Promotion Plan (ref. PREDOC/2015/50) and The European Virtual Institute on Knowledge-based Multifunctional Materials AISBL (KMM-VIN) for the KMM-VIN Research Fellowship (call 2016).

\section{References}

[1] A. Sola et al., Bioactive glass coatings: a review, Surf. Eng. 27 (2011) 560-572

[2] F. Baino et al., Glass-based coatings on biomedical implants: a state-of-the-art review, Biomed Glasses 3 (2017) 1-17

[3] E. Cañas et al., Effect of particle size on processing of bioactive glass powder for atmospheric plasma spraying, J. Eur. Ceram. Soc. 36 (2016) 837-845

[4] G. Bolelli et al., Comparison between suspension plasma sprayed and high velocity suspension flame sprayed bioactive coatings, Surf. Coat. Technol. 280 (2015) 232-249

[5] A. Cattini et al., Suspension plasma sprayed bioactive glass coatings: Effects of processing on microstructure, mechanical properties and in-vitro behaviour, Surf. Coat. Technol. 220 (2013) $52-59$

[6] E. Cañas et al., Bioactive glass coatings by suspension plasma spraying from glycolether-based solvent feedstock, Surf. Coat. Technol. 318 (2017) 190-197

[7] A. Killinger et al., Review of new developments in suspension and solution precursor thermal spray processes, J. Therm. Spray Technol. 20 (2011) 677-695

[8] Y. Xiao et al., Bioactive glass-ceramic coatings synthesized by the liquid precursor plasma spraying process, J. Therm. Spray Technol. 20 (2011) 560-568

[9] M. Vicent et al., Effect of the initial particle size distribution on the properties of suspension plasma sprayed $\mathrm{Al}_{2} \mathrm{O}_{3}-\mathrm{TiO}_{2}$ coatings, Surf. Coat. Technol. 268 (2015) 209-215

[10] A.L.B. Maçon et al., A unified in vitro evaluation for apatite-forming ability of bioactive glasses and their variants, J. Mater. Sci. Mater. Med. 26 (2015) 115-124

[11] M. Gell et al., Thermal barrier coatings made by the solution precursor plasma spray process, J. Therm. Spray Technol. 17 (2008) 124-135

[12] F. Westhauser et al., Micro-computed-tomography-guided analysis of in vitro structural modifications in two types of 45S5 bioactive glass based scaffolds, Mater. 10 (2017) 1341-1354

[13] R.T. Candidato, Development of hydroxyapatite coatings by solution precursor plasma spray process and their microstructural characterization, Surf. Coat. Technol. 318 (2017) $39-49$

[14] S.L. Esteban et al., Bioactive glass coatings for orthopaedic metallic implants, J. Eur. Ceram. Soc. 23 (2003) 2921-2930 